\title{
Two-dimensional speckle-tracking echocardiography assessment of left ventricular remodeling in patients after myocardial infarction and primary reperfusion
}

\author{
Jerzy Liszka' ${ }^{1}$ Maciej Haberka² Zbigniew Tabor $^{3}$, Maciej Finik ${ }^{1}$, Zbigniew Gąsior ${ }^{2}$
}

${ }^{1}$ Department of Cardiology, Multidisciplinary Hospital, Jaworzno, Poland ${ }^{2}$ School of Health Sciences, Medical University of Silesia, Department of Cardiology, Katowice, Poland

${ }^{3} 1^{\text {st }}$ Department of Cardiology, Medical University of Silesia, Katowice, Poland

Submitted: 30 April 2013

Accepted: 5 June 2013

Arch Med Sci 2014; 10, 6: 1091-1100

DOI: 10.5114/aoms.2014.47821

Copyright @ 2014 Termedia \& Banach

\section{Abstract}

Introduction: Left ventricular remodeling (LVR) is the most prognostically important consequence of acute myocardial infarction (AMI). The aim of the study was to assess the value of speckle tracking echocardiography in the prediction of left ventricular remodeling in patients after AMI and primary coronary angioplasty (PCI).

Material and methods: Eighty-eight patients ( $F / M=31 / 57$ patients; 63.6 \pm 11 years old) with coronary artery disease (CAD) and successful PCI were enrolled and divided into group I with ST-elevation myocardial infarction or non-ST elevation myocardial infarction and group II with stable angina pectoris. Conventional and speckle tracking echocardiography was performed 3 days (baseline), 30 days and 90 days after $\mathrm{PCl}$. Patients were divided into 2 groups based on the presence of LVR (increase of LV end-diastolic and/or end-systolic volume $>20 \%$ ) at 3 months follow-up.

Results: At initial presentation, 2 -chamber longitudinal strain $(9.4 \pm 3.5 \%$ vs. $-11.6 \pm 3.6 \%, p<0.04)$ and 4 -chamber transverse strain $(10.4 \pm 8.2 \%$ vs. 15.6 $\pm 8 \%, p<0.003$ ) were lower in the LVR+ group compared to the LVR-group. LV wall motion score index did not differ between the two groups. After 30 days, circumferential apical and basal strain $(-15.58 \pm 8.9 \%$ vs. -25.53 $\pm 8.8 \%, p<0.001 ;-15.02 \pm 5.6$ vs. $-19.78 \pm 6.3, p<0.008)$, radial apical strain $(9.96 \pm 8.4 \%$ vs. $14.15 \pm 5.5 \%, p<0.03)$, 4-chamber longitudinal strain $(-8.7 \pm 5.8 \%$ vs. $-13.47 \pm 3.9 \%, p<0.005)$, 4-chamber transverse strain $(10.5$ $\pm 8.1 \%$ vs. $16.7 \pm 8.3 \%, p<0.03)$, apical rotation $\left(3.84 \pm 2.5^{\circ}\right.$ vs, $5.66 \pm 3.2^{\circ}$, $p<0.04)$ and torsion $\left(6.15 \pm 4.1^{\circ}\right.$ vs. $\left.8.98 \pm 4.6^{\circ}, p<0.03\right)$ were significantly decreased in the LVR+ group compared to the LVR-group. According to ROC analysis, circumferential apical strain $>-15.92 \%$ (sensitivity $93 \%$, specificity $59 \%$, positive predictive value $90 \%$ ) was the most powerful predictor of remodeling after primary $\mathrm{PCl}$ in $\mathrm{AMI}$.

Conclusions: Our results suggest that impaired indices of LV deformation detected 3 days and 30 days after AMI may provide important predictive value in LV remodeling and patients' follow-up.

Key words: acute coronary syndrome, coronary artery disease, echocardiography.

\section{Introduction}

The ongoing improvements in different fields of early diagnosis and successful invasive treatment of acute myocardial infarction (AMI) re-

\author{
Corresponding author: \\ Dr. Jerzy Liszka \\ Department of Cardiology \\ Multidisciplinary Hospital \\ 28 Chełmońskiego St \\ 43-600 Jaworzno, Poland \\ Phone: +48 326164482 \\ Fax: +48 327519844 \\ E-mail: drliszka@tlen.pl, \\ mhaberka@op.pl
}


sulted in continuous reductions in mortality rates associated with early and late complications of AMI. However, even successful percutaneous coronary intervention $(\mathrm{PCl})$ with complete reperfusion does not exclude the risk of left ventricular remodeling (LVR). Left ventricular remodeling is a disadvantageous process resulting in progressive enlargement and changing the shape of the LV cavity with systolic dysfunction as an organ adaptation to the new conditions associated with the tissue infarction. The phenomenon is complex, related to macro- and microscopic changes in the structure and function of cardiomyocytes (scar) [1, 2]. Left ventricular remodeling is well evidenced to be associated with worse outcomes and to predispose to heart failure and death [3]. A detailed description of the processes involved in the remodeling is beyond the scope of the article, and the subject is extensively discussed in the literature.

Despite studies evaluating various clinical factors and routine echocardiographic parameters predisposing to LVR as a consequence of myocardial infarction, there are still gaps in our data, and some of the clinical factors or diagnostic parameters failed to identify patients prone to LVR. Tissue Doppler imaging-derived strain analysis also has drawbacks and may provide limited value in the prediction of left ventricle (LV) structural changes [4]. Speckle tracking echocardiography (STE) is a relatively new echocardiographic method for evaluating and measuring global and regional strain: longitudinal, circumferential, radial and transverse - the precise indices of ventricle function.

Our aim was to evaluate the value of speckle tracking echocardiography in the prediction of left ventricular remodeling in patients after primary coronary angioplasty in acute coronary syndrome.

\section{Material and methods}

Eighty-eight consecutive patients ( $F / M=31 / 57$ patients; $63.6 \pm 11$ years old) with coronary artery disease (CAD) were enrolled in the study and divided into group I with ST-elevation myocardial infarction (STEMI) or non-ST elevation myocardial infarction (NSTEMI) and group II with stable angina pectoris (SAP) (Table I). All the patients (groups I and II) had successful PCl with stent implantation (TIMI 3 flow) prior to enrollment. Patients in group I were followed after the AMI for LVR and prediction factors. Patients with SAP in group II were also followed after the $\mathrm{PCl}$ procedure to as sess potential changes in the routine or STE parameters of LV function within the group resulting from the $\mathrm{PCl}$ and also compare changes in LV function between all AMI patients (independently from LVR) included in group I and reference SAP patients in group II.

All patients included in group I had typical anginal chest pain and significantly increased cardiac markers (CPK - creatine phosphokinase, CPK-MB - creatine phosphokinase-myocardial bound isoenzyme, troponin I). All AMIs were classified as STEMI or NSTEMI with location determination according to electrocardiography (ECG) and echocardiography. All patients included in group II were scheduled for coronary angiography and $\mathrm{PCl}$ according to the European Society of Cardiology (ESC) guidelines. Pharmacotherapy used in all patients also followed the ESC recommendations [5].

The major exclusion criteria were: history of myocardial infarction or any other heart muscle and valvular disease (e.g. heart failure, cardiomyopathies, significant valvular defects, myocarditis) with persistent regional or global LV wall motion abnormalities, any significant general disorder of potential influence on regional or global LV wall motion, significant arrhythmia (including atrial fibrillation and advanced extrasystolic arrhythmia), previous pacemaker or cardioverter-defibrillator implantation, significant heart valve defects, uncompleted reperfusion therapy (coronary artery bypass grafting or repeated $\mathrm{PCl}$ ) and very poor image quality.

Subjects were recruited (from September 2009 to December 2010) and completed the study at the Department of Cardiology at the Medical University of Silesia. The clinical characteristics of

Table I. Infarct-related artery (IRA) in group I and coronary artery requiring $\mathrm{PCI}$ in group II with the number of patients

\begin{tabular}{|c|c|c|c|c|c|c|c|c|c|c|}
\hline & \multicolumn{2}{|c|}{ LAD } & \multicolumn{2}{|c|}{ RCA } & \multicolumn{2}{|c|}{$C x$} & \multicolumn{2}{|c|}{$\mathrm{OM}$} & \multicolumn{2}{|c|}{ D1 } \\
\hline & $M$ & $\mathrm{~F}$ & $M$ & $F$ & $M$ & $\mathrm{~F}$ & $M$ & $\mathrm{~F}$ & $M$ & $\mathrm{~F}$ \\
\hline Group I & 15 & 5 & 21 & 7 & 8 & 3 & 2 & 1 & 1 & 0 \\
\hline$p(\mathrm{M}$ vs. $\mathrm{F})$ & \multicolumn{2}{|c|}{1.0} & \multicolumn{2}{|c|}{1.0} & \multicolumn{2}{|c|}{1.0} & \multicolumn{2}{|c|}{1.0} & \multicolumn{2}{|c|}{-} \\
\hline Group II & 5 & 8 & 2 & 6 & 2 & 1 & 1 & 0 & 1 & 1 \\
\hline$p(\mathrm{M}$ vs. F) & \multicolumn{2}{|c|}{1.0} & \multicolumn{2}{|c|}{0.40} & \multicolumn{2}{|c|}{0.54} & \multicolumn{2}{|c|}{-} & \multicolumn{2}{|c|}{-} \\
\hline$p$ (I vs. II) & \multicolumn{2}{|c|}{0.16} & \multicolumn{2}{|c|}{0.24} & \multicolumn{2}{|c|}{0.54} & \multicolumn{2}{|c|}{1.0} & \multicolumn{2}{|c|}{1.0} \\
\hline
\end{tabular}


the study patients included: medical history (family history, concomitant diseases, prior pharmacotherapy, diet habits and smoking status), physical examination, and standard laboratory tests.

\section{Echocardiography}

Two-dimensional (2D) transthoracic echocardiography (TTE) was performed in all patients on admission to the hospital (not included in the study results) and then: 3 days (baseline examination - "0"), 30 days ("30") and 90 days ("90") after the $\mathrm{PCl}$ procedure. Both 2D-TTE with the routine parameters and 2D-STE were ECG-gated and performed during each of the visits. All the patients were examined in the left lateral decubitus position using a 3.5 MHz transducer in the standard views: parasternal (long axis and short axis - basal, midventricular, apical level) and apical (two-, three- and four-chamber). The images and video clips containing cine-loop format were recorded by one expert (90-103 frames per second) and then analyzed offline independently by two experts. All the measurements used for statistical analysis were averaged from three consecutive beats. The LV was divided into 17 segments, evaluated and scored (1 - normal, 2 - hypokinesis, 3 - akinesis, 4 - dyskinesis, 5 - aneurysmal), and the global wall motion score index (WMSI) was calculated for each TTE examination [6]. The LV volumes and ejection fraction (EF) were determined using the modified Simpson biplane technique.

The LV remodeling was defined as $\geq 20 \%$ increase in LV end-diastolic volume (EDV) and/or LV end-systolic volume (ESV) at 3-month follow-up compared with the baseline examination $[7,8]$.

Myocardial tissue deformation was analyzed offline using commercially available software (STE Toshiba) using recorded 2D gray-scale images. End-systole was defined as an aortic valve closure in the apical 5-chamber view. The endocardial borders were traced manually in an end-systole with the myocardium in the region of interest (ROI). Then the position and the width of the ROI were optimized. The software analyzed the speckles within the myocardium and calculated segmental strain. The peak longitudinal strain and peak transverse strain were calculated as the average of peak values for each of the segments in the apical 2-chamber and 4-chamber views (longitudinal and transverse strain) and parasternal short axis views at three levels (transverse and circumferential strain). Rotation was estimated using basal and apical short axis views: the clockwise rotation was defined as negative and the counterclockwise rotation was defined as positive when determined from the apical aspect. The LV twist was defined as the absolute difference between apical and basal rotation values.
The values of interobserver and intraobserver variability in analysis of patients' recordings were $92 \%$ and $95 \%$.

\section{Statistical analysis}

The strain values are expressed in percentages and rotation is expressed in degrees. All results presented in the text and tables are expressed as means \pm standard deviation or number and percentage. A value of $p<0.05$ was considered statistically significant. The results' normal distribution was analyzed with the Kolmogorov-Smirnov test. Levene and Brown-Forsythe tests were used to verify the homogeneity of variance in the group. Baseline clinical parameters and the results of diagnostic tests were compared using $t$-tests for normally distributed continuous variables (Student's $t$-test); in case of abnormal distribution, the Mann-Whitney $U$ test was used. Pearson's test with Yates' correction was used for qualitative variables. The Kruskal-Wallis test was used to assess the variables within several subgroups. Predictive value of particular parameters was determined using the univariate logistic regression method for estimation by Rosenbrock and the quasi-Newton method. The receiver operating characteristic (ROC) curves were determined with Statistica 8.0 software. Correlation analysis was performed using Pearson's test (normal distribution) or Spearman's rank correlation.

\section{Results}

Seventy consecutive patients with STEMI or NSTEMI (group I) and 30 consecutive patients with SAP (group II) were screened with the eligibility criteria. Twelve patients were not included in the study because of the following reasons: prior heart failure (4 patients), prior myocardial infarction with LV scar (3 patients), poor image quality (1 patient - obesity, 1 patient - emphysema), arrhythmia (2 patients - atrial fibrillation, 1 patient - significant extrasystole). The feasibility of LV segment analysis with STE was 93\% for the longitudinal strain and $88 \%$ for the transverse, circumferential strain and torsion analysis. All the subjects completed the follow-up and none of the patients suffered from the clinical endpoint, including cardiovascular death, acute coronary syndrome, unstable angina pectoris, acute heart failure, $\mathrm{PCl}$ or re-hospitalization. The final diagnosis with the number of patients (males/females) in group I was as follows: STEMI anterior wall (9/3), STEMI inferior wall (17/8), STEMI lateral wall (2/0) and NSTEMI (19/5). Table I presents the infarct-related artery (IRA) in group I and coronary artery requiring $\mathrm{PCl}$ in group $\|$ with the number of patients. 
Group I (AMI) and II (SAP) echocardiographic characteristics

The baseline examination revealed significantly larger LV EDV, lower LV EF and higher WMSI in group I (AMI) compared to group II (SAP). The echocardiography performed 90 days after $\mathrm{PCl}$ showed significantly larger LV EDV and ESV, lower EF and higher WMSI in patients with AMI compared to patients with SAP. There were no differences in mitral regurgitation severity between the groups at both time points. The routine and speckle-tracking echocardiography parameters in groups I and II are presented in Table II.

\section{Group I patients' echocardiography characteristics and left ventricular remodeling prediction}

During a 3-month follow-up, LV remodeling occurred in 17 patients (27\%) in group I. The rate of LVR was independent of age and the type or location of MI (Table III). Patients in the R+ group had significantly lower EF (52.9 $\pm 8.4 \%$ vs. $58.5 \pm 8.6 \%$; $p<0.02)$ and larger ESV (38.5 $\pm 13.7 \mathrm{ml}$ vs. 49.9 $\pm 26.4 \mathrm{ml} ; p<0.001)$ at the baseline and also had lower EF $(50.53 \pm 7.0 \%$ vs. $61.20 \pm 7.7 ; p<0.001)$ and larger ESV $(67.53 \pm 25.3 \mathrm{ml}$ vs. $36.43 \pm 13.5 \mathrm{ml})$ and EDV (142 \pm 41.06 vs. $94.85 \pm 25.8$; $p<0.001)$ at the 3-month follow-up compared to the $\mathrm{R}$ - group (Table IV). However, neither the baseline nor the 3-month follow-up WMSI differentiated the two groups. Baseline STE revealed significantly lower mean longitudinal global strain in apical two-chamber view $(-9.4 \pm 3.5 \%$ vs. $-11.6 \pm 3.6 \%$; $p<0.004)$ and mean transverse strain in apical four-chamber view (10.4 $\pm 8.2 \%$ vs. $15.6 \pm 8 \%$; $p<0.03$ ) in patients with LV remodeling compared to the R-group. However, 30 days and 90 days after the AMI there were several significant differences between the groups (Tables IV, V).

\section{Regression and receiver operating characteristic curve analysis}

Univariate analysis showed that lower EF, larger ESV and lower longitudinal strain in 4-chamber (sLa4 0) and 2-chamber view (sLa2 0), transverse strain in 4-chamber view (sTa4 0) and circumferential strain in basal segments obtained at the baseline (sCbas 0 ) were significantly related to LV remodeling. Moreover, the following parameters obtained 1 month after $\mathrm{Ml}$ had predictive value: circumferential strain in apical and basal segments (sCapex 30, sCbas 30), radial strain in

Table II. Routine and speckle tracking echocardiography (STE) in group I and II patients at baseline and 90 days after $\mathrm{PCl}$

\begin{tabular}{|c|c|c|c|c|c|c|}
\hline \multirow[t]{2}{*}{ Parameter } & \multicolumn{3}{|c|}{ Baseline visit } & \multicolumn{3}{|c|}{ Control visit 90 days after $\mathrm{PCl}$} \\
\hline & Group I & Group II & Value of $p$ & Group I & Group II & Value of $p$ \\
\hline LVEDV [ml] & $98.37 \pm 26.7$ & $85.36 \pm 23.8$ & $<0.05$ & $107.57 \pm 36.9$ & $87.36 \pm 22.7$ & 0.02 \\
\hline LVESV [ml] & $41.56 \pm 18.5$ & $33.20 \pm 16.0$ & 0.05 & $44.83 \pm 22.16$ & $32.44 \pm 16.9$ & 0.02 \\
\hline EF (\%) & $56.98 \pm 8.9$ & $62.88 \pm 7.5$ & $<0.01$ & $58.32 \pm 8.9$ & $64.16 \pm 6.4$ & $<0.01$ \\
\hline WMSI & $1.16 \pm 0.2$ & $1.02 \pm 0.1$ & $<0.01$ & $1.2 \pm 0.3$ & $1.02 \pm 0.1$ & $<0.01$ \\
\hline sCapex (\%) & $-22.77 \pm 10.5$ & $-26.81 \pm 10.22$ & 0.10 & $-22.85 \pm 9.9$ & $-29.03 \pm 8.6$ & $<0.01$ \\
\hline sCbas (\%) & $-16.91 \pm 7.2$ & $-20.46 \pm 6.7$ & 0.04 & $-18.50 \pm 6.4$ & $-20.88 \pm 6.3$ & 0.12 \\
\hline sRapex (\%) & $11.89 \pm 7.0$ & $13.79 \pm 7.8$ & 0.27 & $14.15 \pm 5.5$ & $13.02 \pm 6.6$ & 0.09 \\
\hline sRbas (\%) & $19.00 \pm 10.3$ & $20.85 \pm 6.9$ & 0.40 & $19.86 \pm 8.1$ & $24.16 \pm 9.7$ & 0.04 \\
\hline sLa2 (\%) & $-11.01 \pm 3.7$ & $-12.73 \pm 2.1$ & 0.03 & $-11.80 \pm 4.2$ & $-12.21 \pm 2.9$ & 0.66 \\
\hline sLa4 (\%) & $-11.36 \pm 4.3$ & $-12.63 \pm 2.9$ & 0.18 & $12.18 \pm 4.9$ & $-14.23 \pm 3.6$ & 0.06 \\
\hline sTa2 (\%) & $14.02 \pm 7.5$ & $14.03 \pm 6.5$ & 0.99 & $14.28 \pm 8.2$ & $17.87 \pm 9.1$ & 0.08 \\
\hline sTa4 (\%) & $14.18 \pm 8.3$ & $14.79 \pm 7.7$ & 0.75 & $15.00 \pm 8.6$ & $15.69 \pm 7.5$ & 0.72 \\
\hline Rot apex $\left[^{\circ}\right]$ & $6.43 \pm 3.7$ & $5.52 \pm 3.8$ & 0.31 & $5.17 \pm 3.1$ & $7.38 \pm 3.9$ & 0.01 \\
\hline Rot bas $\left[^{\circ}\right]$ & $-2.83 \pm 2.1$ & $-3.78 \pm 2.6$ & 0.08 & $-3.58 \pm 2.6$ & $-5.22 \pm 3.5$ & 0.02 \\
\hline Torsion [ํ] & $8.74 \pm 5.0$ & $9.38 \pm 5.5$ & 0.73 & $8.22 \pm 4.3$ & $12.24 \pm 6.1$ & $<0.01$ \\
\hline
\end{tabular}

PCI - percutaneous coronary intervention, LVEDV - left ventricular end diastolic volume, LVESV - left ventricular end systolic volume, $E F$ - ejection fraction, WMSI - wall motion score index, $S$ - strain, $C$ - circumferential, $R$ - radial, $L$ - longitudinal, $T$ - transverse, apex apical segments, bas - basal segments, a2 - apical 2-chamber view, a4 - apical 4-chamber view, Rot-rotation. 
Table III. Group I: patients with and without left ventricle remodeling R (+) vs. R (-)

\begin{tabular}{|c|c|c|c|}
\hline Parameter & Group I R (+) & Group I R (-) & Value of $p$ \\
\hline \multicolumn{4}{|l|}{ Baseline characteristics: } \\
\hline Age & $61.48 \pm 12.1$ & $64.65 \pm 11.2$ & 0.35 \\
\hline Females/males & $2(3 \%) / 15(24 \%)$ & $14(22 \%) / 32(51 \%)$ & 0.19 \\
\hline Smoking & $8(47 \%)$ & $23(50 \%)$ & 0.12 \\
\hline Diabetes & $5(29 \%)$ & $9(19 \%)$ & 0.49 \\
\hline Arterial hypertension & $11(65 \%)$ & $31(67 \%)$ & 0.84 \\
\hline \multicolumn{4}{|l|}{ Pharmacotherapy: } \\
\hline Acetylsalicylic acid & 17 (100\%) & $46(100 \%)$ & 1.0 \\
\hline Clopidogrel & 17 (100\%) & $46(100 \%)$ & 1.0 \\
\hline$\beta$-Blockers & 17 (100\%) & $46(100 \%)$ & 1.0 \\
\hline ACE-I/ARB & $16(94 \%)$ & $42(91 \%)$ & 0.72 \\
\hline Statins & 17 (100\%) & $46(100 \%)$ & 1.0 \\
\hline \multicolumn{4}{|l|}{ Infarct-related artery: } \\
\hline Left anterior descending & 4 & 17 & 0.37 \\
\hline Right coronary artery & 8 & 20 & 1.0 \\
\hline Circumflex artery & 5 & 9 & 0.49 \\
\hline \multicolumn{4}{|l|}{ Coronary artery disease: } \\
\hline 1 -vessel CAD & $3(18 \%)$ & $18(39 \%)$ & 0.14 \\
\hline 2-vessel CAD & $8(47 \%)$ & $13(28 \%)$ & 0.23 \\
\hline 3-vessel CAD & $6(35 \%)$ & $15(33 \%)$ & 1.0 \\
\hline STEMI anterior & 3 & 8 & 1.0 \\
\hline STEMI inferior & 7 & 20 & 1.0 \\
\hline STEMI lateral & 1 & 1 & 0.47 \\
\hline NSTEMI & 6 & 17 & 1.0 \\
\hline Killip class & $1.17 \pm 0.4$ & $1.41 \pm 0.4$ & 0.31 \\
\hline Time to reperfusion & $15 \pm 21.5$ & $8 \pm 12.6$ & 0.03 \\
\hline TIMI 3 & $17(100 \%)$ & $46(100 \%)$ & 1.0 \\
\hline Stent implantation & 17 (100\%) & $46(100 \%)$ & 1.0 \\
\hline
\end{tabular}

CAD - coronary artery disease, ACE-I - angiotensin-converting enzyme inhibitors, ARB - angiotensin receptor blockers, STEMI-ST elevation myocardial infarction, TIMI - thrombolysis in myocardial infarction.

apex (sRapex 30) and basal segments (sRbas 30), longitudinal strain in 4-chamber view (sLa4 30), transverse strain in 4-chamber view (sTa4 30), apical rotation (rotapex 30) and torsion (Table VI). Multivariable analysis revealed that decreased EF at baseline and decreased SCapex and SLa4 obtained 30 days after the $P C I$ are related to LVR (Table VI). Moreover, the receiver operating characteristic curve (ROC) analysis showed cutoff values for STE parameters predicting LVR, presented in Table VII.

\section{Discussion}

Our results suggest that most types of deformation, including rotation and torsion, reveal different degrees of impairment at the baseline or 30 days after the AMI in LVR patients. We found impaired basal or apical segments' circumferential, longitudinal or transverse strain parameters in the LVR(+) patients compared to the $\operatorname{LVR}(-)$ group. Hence, some strain parameters were significantly different no sooner than 30 days after the AMI, which is an expected consequence of progressive cardi- 
Table IV. Speckle tracking echocardiography (STE) in group I R (-) and group I R (+) patients at baseline and 90 days after $\mathrm{PCl}$

\begin{tabular}{|c|c|c|c|c|c|c|}
\hline \multirow[t]{2}{*}{ Parameter } & \multicolumn{3}{|c|}{ Baseline visit } & \multicolumn{3}{|c|}{ Control visit 90 days after $\mathrm{PCl}$} \\
\hline & Group R (-) & Group R (+) & Value of $p$ & Group R (-) & Group R (+) & Value of $p$ \\
\hline LVEDV [ml] & $95.50 \pm 26.8$ & $106.12 \pm 32.9$ & 0.19 & $94.85 \pm 25.8$ & $142.00 \pm 41.06$ & $<0.01$ \\
\hline LVESV [ml] & $38.46 \pm 13.7$ & $49.94 \pm 26.4$ & $<0.03$ & $36.43 \pm 13.5$ & $67.53 \pm 25.3$ & $<0.01$ \\
\hline EF (\%) & $58.50 \pm 8.6$ & $52.88 \pm 8.4$ & $<0.02$ & $61.20 \pm 7.7$ & $50.53 \pm 7.0$ & $<0.01$ \\
\hline WMSI & $1.14 \pm 0.2$ & $1.18 \pm 0.2$ & 0.41 & $1.09 \pm 0.1$ & $1.32 \pm 0.3$ & $<0.05$ \\
\hline MR grade & $1.89 \pm 1.7$ & $2.02 \pm 2.2$ & 0.89 & $1.72 \pm 1.8$ & $3.19 \pm 2.3$ & $<0.01$ \\
\hline sCapex (\%) & $-23.11 \pm 10.6$ & $-21.83 \pm 10.3$ & 0.67 & $-25.53 \pm 8.85$ & $-15.58 \pm 8.9$ & $<0.01$ \\
\hline sCbas (\%) & $-17.85 \pm 7.4$ & $-14.36 \pm 5.1$ & 0.09 & $-19.78 \pm 6.3$ & $-15.02 \pm 5.6$ & $<0.01$ \\
\hline sRapex (\%) & $11.72 \pm 6.8$ & $12.34 \pm 7.8$ & 0.76 & $14.15 \pm 5.5$ & $9.96 \pm 8.4$ & $<0.03$ \\
\hline sRbas (\%) & $20.15 \pm 10.6$ & $15.92 \pm 9.2$ & 0.15 & $20.83 \pm 8.3$ & $17.23 \pm 7.1$ & 0.12 \\
\hline sLa2 (\%) & $-11.61 \pm 3.6$ & $-9.40 \pm 3.7$ & $<0.04$ & $-12.2 \pm 4.0$ & $-10.64 \pm 4.5$ & 0.18 \\
\hline sLa4 (\%) & $-11.99 \pm 4.2$ & $-9.65 \pm 4.3$ & 0.06 & $-13.47 \pm 3.9$ & $-8.71 \pm 5.8$ & $<0.01$ \\
\hline sTa2 (\%) & $14.81 \pm 6.9$ & $11.88 \pm 8.7$ & 0.17 & $14.37 \pm 7.9$ & $14.03 \pm 9.3$ & 0.89 \\
\hline sTa4 (\%) & $15.58 \pm 8.0$ & $10.36 \pm 8.2$ & $<0.03$ & $16.67 \pm 8.3$ & $10.48 \pm 8.1$ & $<0.03$ \\
\hline Rot apex $\left[^{\circ}\right]$ & $6.92 \pm 3.9$ & $5.08 \pm 2.6$ & 0.08 & $5.66 \pm 3.2$ & $3.84 \pm 2.5$ & $<0.04$ \\
\hline Rot bas $\left[^{\circ}\right]$ & $-2.79 \pm 1.9$ & $-2.93 \pm 2.7$ & 0.8 & $-3.71 \pm 2.8$ & $-3.21 \pm 1.9$ & 0.5 \\
\hline Torsion [0] & $9.09 \pm 5.4$ & $7.79 \pm 5.3$ & 0.3 & $8.98 \pm 4.6$ & $6.15 \pm 4.1$ & $<0.03$ \\
\hline
\end{tabular}

LVEDV - left ventricular end diastolic volume, LVESV - left ventricular end systolic volume, EF - ejection fraction, WMSI - wall motion score index, $M R$ - mitral regurgitation, $s$ - strain, $C$ - circumferential, $R$ - radial, $L$ - longitudinal, $T$ - transverse, apex - apical segments, bas basal segments, a2 - apical 2-chamber view, a4-apical 4-chamber view, Rot-rotation.

Table V. Speckle tracking echocardiography (STE) in group I R (-) and group I R (+) patients at baseline and 30 days after $\mathrm{PCl}$

\begin{tabular}{|c|c|c|c|c|c|c|}
\hline \multirow[t]{2}{*}{ Parameter } & \multicolumn{3}{|c|}{ Baseline visit } & \multicolumn{3}{|c|}{ Control visit 30 days after $\mathrm{PCl}$} \\
\hline & Group R (-) & Group R (+) & Value of $p$ & Group R (+) & Group R (-) & Value of $p$ \\
\hline sCapex (\%) & $-23.11 \pm 10.6$ & $-21.83 \pm 10.3$ & 0.67 & $-25.53 \pm 8.85$ & $-15.58 \pm 8.9$ & $<0.01$ \\
\hline sCbas (\%) & $-17.85 \pm 7.4$ & $-14.36 \pm 5.1$ & 0.09 & $-19.78 \pm 6.3$ & $-15.02 \pm 5.6$ & $<0.01$ \\
\hline sRapex (\%) & $11.72 \pm 6.8$ & $12.34 \pm 7.8$ & 0.76 & $14.15 \pm 5.5$ & $9.96 \pm 8.4$ & $<0.03$ \\
\hline sRbas (\%) & $20.15 \pm 10.6$ & $15.92 \pm 9.2$ & 0.15 & $20.83 \pm 8.3$ & $17.23 \pm 7.1$ & 0.12 \\
\hline sLa2 (\%) & $-11.61 \pm 3.6$ & $-9.40 \pm 3.5$ & $<0.04$ & $-12.2 \pm 4.0$ & $-10.64 \pm 4.5$ & 0.18 \\
\hline sLa4 (\%) & $-11.99 \pm 4.2$ & $-9.65 \pm 4.3$ & 0.06 & $-13.47 \pm 3.9$ & $-8.71 \pm 5.8$ & $<0.01$ \\
\hline sTa2 (\%) & $14.81 \pm 6.9$ & $11.88 \pm 8.7$ & 0.17 & $14.37 \pm 7.9$ & $14.03 \pm 9.3$ & 0.89 \\
\hline sTa4 (\%) & $15.58 \pm 8.0$ & $10.36 \pm 8.2$ & $<0.03$ & $16.67 \pm 8.3$ & $10.48 \pm 8.1$ & $<0.03$ \\
\hline Rot apex $\left[^{\circ}\right]$ & $6.92 \pm 3.9$ & $5.08 \pm 2.6$ & 0.08 & $5.66 \pm 3.2$ & $3.84 \pm 2.5$ & $<0.04$ \\
\hline Rot bas [ $\left.{ }^{\circ}\right]$ & $-2.79 \pm 1.9$ & $-2.93 \pm 2.7$ & 0.8 & $-3.71 \pm 2.8$ & $-3.21 \pm 1.9$ & 0.50 \\
\hline Torsion [ $\left.{ }^{\circ}\right]$ & $9.09 \pm 5.4$ & $7.79 \pm 5.3$ & 0.3 & $8.98 \pm 4.6$ & $6.15 \pm 4.1$ & $<0.03$ \\
\hline
\end{tabular}

$s$ - strain, C - circumferential, $R$ - radial, $L$ - longitudinal, $T$ - transverse, apex - apical segments, bas - basal segments, a2 - apical 2-chamber view, a4-apical 4-chamber view, Rot-rotation.

ac remodeling. Multivariate regression analysis showed that lower baseline EF and apical circumferential strain and longitudinal 4-chamber view strain obtained 30 days after the AMI were independently related to LVR. Therefore, STE performed 1 month after the AMI also provides important val- 
Table VI. Univariate and multivariate logistic regression analysis: baseline echocardiographic parameters and left ventricle remodeling

\begin{tabular}{|c|c|c|c|c|c|c|}
\hline \multirow[t]{2}{*}{ Parameter } & \multicolumn{3}{|c|}{ Univariate } & \multicolumn{3}{|c|}{ Multivariate } \\
\hline & OR & $95 \% \mathrm{Cl}$ & Value of $p$ & OR & $95 \% \mathrm{Cl}$ & Value of $p$ \\
\hline LVESV & 0.97 & $0.94 ; 0.99$ & 0.01 & & & \\
\hline EF & 1.09 & $1.02 ; 1.16$ & $<0.01$ & 0.89 & $0.8 ; 0.98$ & $<0.01$ \\
\hline sCbas & 1.10 & $1.01 ; 1.20$ & 0.03 & & & \\
\hline sLa2 & 0.79 & $0.66 ; 0.94$ & $<0.01$ & & & \\
\hline sLa4 & 1.18 & $1.02 ; 1.37$ & 0.02 & & & \\
\hline sTa4 & 1.09 & $1.00 ; 1.18$ & 0.03 & & & \\
\hline sCapex 30 & 1.17 & $1.07 ; 1.27$ & $<0.01$ & 0.88 & $0.79 ; 0.97$ & $<0.01$ \\
\hline sCbas 30 & 1.15 & $1.04 ; 1.28$ & $<0.01$ & & & \\
\hline sRapex 30 & 1.13 & $1.02 ; 1.24$ & 0.01 & & & \\
\hline sRbas 30 & 1.08 & $1.00 ; 1.16$ & 0.04 & & & \\
\hline sLa4 30 & 1.29 & $1.10 ; 1.50$ & $<0.01$ & 0.82 & $0.68 ; 0.96$ & 0.01 \\
\hline sTa4 30 & 1.10 & $1.02 ; 1.20$ & 0.01 & & & \\
\hline Rot apex 30 & 1.32 & $1.06 ; 1.66$ & 0.01 & & & \\
\hline Torsion 30 & 0.84 & $0.73 ; 0.95$ & $<0.01$ & & & \\
\hline WMSI & 0.10 & $0.007 ; 1.54$ & 0.09 & & & \\
\hline
\end{tabular}

$O R$ - odds ratio, $C I$ - confidence interval, LVESV - left ventricular end systolic volume, EF- ejection fraction, $s$ - strain, $C$ - circumferential, $R$ - radial, $L$ - longitudinal, $T$-transverse, apex-apical segments, bas - basal segments, a2 - apical 2-chamber view, a4-apical 4-chamber view, Rot-rotation

Table VII. Echocardiographic parameters obtained at the baseline visit predicting left ventricle remodeling in the 3-month follow-up

\begin{tabular}{|lcccccc|}
\hline Parameter & Value & Sensitivity & Specificity & ACC & PPV & NPV \\
\hline SLa4 0 & -9.98 & 0.803 & 0.647 & 0.773 & 0.905 & 0.440 \\
\hline SLa2 0 & -7.98 & 0.94 & 0.35 & 0.83 & 0.86 & 0.60 \\
\hline sTa4 0 & 4.03 & 0.97 & 0.35 & 0.85 & 0.86 & 0.75 \\
\hline sCapex 30 & -15.92 & 0.930 & 0.588 & 0.864 & 0.904 & 0.667 \\
\hline sLa4 30 & -7.86 & 0.944 & 0.529 & 0.864 & 0.893 & 0.692 \\
\hline
\end{tabular}

sLa4 0 - baseline longitudinal 4-chamber strain, sCapex 30 - apical segments circumferential strain 30 days after AMI, sLa4 30 longitudinal 4-chamber strain 30 days after AMI, ACC - accuracy, PPV-positive predictive value, NPV-negative predictive value.

ue in LVR prediction. Both examinations revealed that decreased torsion observed in group I compared to group II was related mainly to lower rotation at the basal segments $\mathrm{R}(-)$ or apical segments $R(+)$. The ROC analysis suggests that at baseline, 4-chamber transverse strain (sTa4) shows the best sensitivity with lower specificity, while 4-chamber longitudinal strain (sLa4) reveals better specificity, but lower sensitivity. STE performed 30 days after the $\mathrm{PCl}$ shows the highest sensitivity, specificity and accuracy for apical segments' circumferential strain and 4-chamber longitudinal strain.

Our study aimed to assess the additive value of STE to conventional echocardiography in the prediction of negative structural changes. We evaluated several STE parameters obtained not only at the baseline and 3 months or later after the $\mathrm{AMI}$ as in most other available observations, but also at a third time point (1 month). Our results may also help to select STE indices and the time after the AMI to best predict LVR. There are a few studies suggesting additive value of various STE parameters in predicting LVR. D'Andrea et al. found that the averaged strain obtained in all LV segments (global longitudinal strain) is a reliable predictor of LVR $(\geq 15 \%$ increase in LV EDV at 6 months after AMI) with a sensitivity of $84.8 \%$ and specificity of $87.8 \%$ [9]. Hung et al. related 
4-chamber and 2-chamber global peak longitudinal strain and parasternal short-axis global circumferential strain to the LVR at 20-month follow-up. Although both types of strain were associated with clinical outcomes, only circumferential analysis was found to be predictive of LVR ( $\geq 15 \%$ increase in LV ESV assessed at 20-month follow-up) [10]. Zaliaduonyte-Peksiene et al. also confirmed a predictive role of global longitudinal strain in prediction of LVR following AMI $(\geq 15 \%$ increase in LV EDV at 4-month follow-up) [11]. Both Nucifora et al. [12] and Jang et al. [3] reported that impaired LV torsion may help predict LVR assessed 6 months after the AMI and defined as $\geq$ $15 \%$ or $\geq 20 \%$ increase in LV EDV.

Longitudinal strain reflects subendocardial longitudinal layer of heart muscle contraction, which is usually impaired at the early phase of ischemia, while circumferential and radial strain or apex rotation may stay still normal and will be impaired in the following stages [13-15]. Chan et al. suggest that a significant decrease in circumferential strain is observed when infarction transmurality is more than 75\% [16]. Therefore, decreased circumferential strain may be an independent cardiovascular risk factor providing more important value than longitudinal strain $[12,17]$.

In our study, the rate of LV remodeling was $27 \%$, which is comparable to other observations [1820]. Although various criteria and definitions are found in the literature data, we used at least $20 \%$ increase in left ventricular end diastolic volume (LVEDV) and/or ESV assessed 3 months after the MI [18, 21, 22]. At baseline, we found increased left ventricular end systolic volume (LVESV) and decreased EF with no significant differences in LVEDV or WMSI between R+ and R-groups. However, there are studies where increased WMSI and larger ischemia predicted LVR [20, 21, 23]. Anterior wall infarction with large ischemia and the LAD as the ischemia-related coronary artery are the most common risk factors for $\operatorname{LVR}[20,21$, $24,25]$. However, some post-MI groups did not provide similar observations [18, 26, 27]. Masci et al. found that baseline AMI area assessed in the cardiovascular magnetic resonance, but not its location (anterior vs. non-anterior $\mathrm{MI}$ ), predicted LVR in the 4-month follow-up [28]. In our study, the most frequent IRA was RCA (41\%) and inferior wall $\mathrm{MI}$, but we did not observe any relations between the MI location and LVR. Most, but not all, patients with LVR had STEMI (60\%), though NSTEMI diagnosis does not exclude transmural infarction. There are also findings other than LV volume or echocardiographic EF suggesting increased risk of LVR, including: significant diastolic dysfunction [20], restrictive mitral filling [29], LV dyssynchrony [30] and myocardial perfusion in contrast-enhanced echocardiography [31]. Mitral valve regurgitation is common in patients with $\mathrm{MI}$ (15-64\%), constitutes an independent risk factor for cardiovascular mortality, and may be a predictive risk factor or even may accelerate LVR [32, 33]. Hence, both groups in our study were comparable in baseline mitral regurgitation severity. There was a significantly longer mean time from first symptoms occurrence to reperfusion in patients with LVR, which was also reported in some $[23,34,35]$ but not all other studies [21, 24, 29, 36]. The first symptoms-reperfusion time consists of three parts: time to first medical contact, time to medical center, and door to balloon time. The relatively long overall time to reperfusion found in our patients was mainly due to their unnecessary emergency call delay despite clear symptoms or ambiguous and atypical general symptoms, which patients primarily did not associate with their cardiovascular system (e.g. gastrointestinal-like symptoms in inferior wall ischemia). Still, an early and successful reperfusion of the IRA seems to be the major factor predisposing to unfavorable changes in LV structure and function [20,37]. The LVR occurring despite successful reperfusion may be a consequence of a lower rate of viable cardiomyocytes in the area of infarction unable to prevent adverse remodeling [21]. The longitudinal strain and strain rate in the post-MI patients may reflect the rate of viable cardiomyocytes as observed in the early dobutamine stress test [38, 39]. Park et al. found that longitudinal strain below $-10.2 \%$ assessed early in post-MI patients (successful reperfusion) may predict nonviable myocardium in remodeled LV (sensitivity 90.9\% and specificity $81.8 \%$ ) [40].

The main limitation of our study is a relatively small sample with the predominance of STEMI (vs. NSTEMI) and inferior wall location. We did our best to obtain only high-quality echocardiographic images, but still some suboptimal image quality affecting strain measurements may have occurred, as in all studies that used the STE method.

In conclusion, LV remodeling is found in a relatively significant number of patients after AMI with reperfusion therapy and is strongly associated with the time to reperfusion and lower EF at discharge. Our results suggest that STE performed early at the discharge and 1 month after the AMI reveals LV strain impairment and may provide important predictive value in identifying patients at high risk for LVR. The most important STE predictor of LVR was apical circumferential strain obtained 30 days after the AMI. Early LVR suspicion enables us to select individuals requiring more detailed and frequent follow-up or aggressive secondary prevention. 


\section{References}

1. Cohn JN, Ferrari R, Sharpe N, et al. Cardiac remodeling - concepts et clinical implications: a consensus paper from an international forum on cardiac remodeling. J Am Coll Cardiol 2000; 35: 269-82.

2. McKay RG, Pfeffer MA, Pasternak RC. Left ventricular remodeling after myocardial infarction: a corollary to infarct expansion. Circulation 1986; 74: 693-702.

3. Jang JY, Woo JS, Kim WS, et al. Serial assessment of left ventricular remodeling by measurement of left ventricular torsion using speckle tracking echocardiography in patients with acute myocardial infarction. Am J Cardiol 2010; 106: 917-23.

4. Wilinski J, Czarnecka D, Wojciechowska W, et al. Baseline tissue Doppler imaging-derived echocardiographic parameters and left ventricle reverse remodeling following cardiac resynchronization therapy introduction. Arch Med Sci 2011; 7: 813-22.

5. Wijns W, Kolh P, Danchin N, et al. Guidelines on myocardial revascularization. The Task Force on Myocardial Revascularization of the European Society of Cardiology (ESC) and the European Association for Cardio-Thoracic Surgery (EACTS). Eur Heart J 2010; 31: 2501-55.

6. Lang RM, Bierig M, Devereux RB, et al. Recommendations for chamber quantification: A Report from the American Society of Echocardiography's Guidelines and Standards Committee and the Chamber Ouantification Writing Group, Developed in Conjunction with the European Association of Echocardiography, a Branch of the European Society of Cardiology. J Am Socechocardiogr 2005; 18: 1440-63.

7. Rizzello V, Poldermans D, Boersma E, et al. Opposite patterns of left ventricular remodeling after coronary revascularization in patients with ischemic cardiomyopathy. Role of myocardial viability. Circulation 2004; 110 : 2383-8.

8. Sarai M, Biswas S, Toyama $\mathrm{H}$, et al. Ventricular remodeling following acute myocardial infarction and its correlation with discordant perfusion and fatty acid metabolism. J Nucl Med 2009; 50: 1136.

9. D'Andrea A, Cocchia R, Caso P, et al. Global longitudinal speckle-tracking strain is predictive of left ventricular remodeling after coronary angioplasty in patients with recent non-ST elevation myocardial infarction. Int I Cardiol 2011; 153: 185-91.

10. Hung C, Verma A, Uno H, et al. Longitudinal and circumferential strain rate, left ventricular remodeling, and prognosis after myocardial infarction. J Am Coll Cardiol 2010; 56: 1812-22.

11. Zaliaduonyte-Peksiene D, Vaskelye JJ, Mizariene V, Jurkevicius R, Zaliunas R. Does longitudinal strain predict left ventricular remodeling after myocardial infarction? Echocardiography 2012; 29: 419-27.

12. Nucifora G, Marsan NA, Bertini M, et al. Reduced left ventricular torsion early after myocardial infarction is related to left ventricular remodeling. Circ Cardiovasc Imaging 2010; 3: 433-42.

13. Geyer H, Caracciolo G, Abe H, et al. Assesment of myocardial mechanics using speckle tracking echocardiography: fundaments and clinical applications. J Am Soc Echocardiogr 2010; 23: 351-69.

14. Horie H, Takahashi M, Minai K. Long-term beneficial effect of late reperfusion for acute anterior myocardial infarction with percutaneous transluminal coronary angioplasty. Circulation 1998; 98: 2377-82.

15. Song ZZ. Global longitudinal strain by two-dimensional speckle tracking echocardiography is closely related to myocardial infarct size in chronic ischemic heart disease. Clin Sci 2007; 113: 393-5.

16. Chan J, Hanekom L, Wong C, et al. Differentiation of subendocardial and transmural infarction using two-dimensional strain rate imaging to assess short-axis and long-axis myocardial function. JACC 2006; 48: 2026-33.

17. Peng Y, Popović ZB, Sopko N, et al. Speckle tracking echocardiography in the assessment of mouse models of cardiac dysfunction. AJP Heart 2009; 297: 811-20.

18. Ko JS, Jeong MH, Lee MG, et al. Left ventricular dyssynchrony after acute myocardial infarction is a powerful indicator of left ventricular remodeling. Korean Circ J 2009; 39: 236-42.

19. Araszkiewicz A, Lesiak M, Grajek S, et al. Relationship between tissue reperfusion and postinfatction left ventricular remodeling in patients with anterior wall myocardial infarction treated with primary coronary angioplasty. Kardiol Pol 2006; 64: 383-8.

20. Łoboz-Grudzień K, Kowalska A, Brzezińska B. Early predictors of adverse left ventricular remodeling after myocardial infarction treated by primary angioplasty. Cardiol I 2007; 14: 238-46.

21. Bolognese L, Neskovic A, Parodi G. Left ventricular remodeling after primary coronary angioplasty. Circulation 2002; 106: 2351-7.

22. Jang JY, Woo JS, Kim WS, et al. Serial assesment of left ventricular remodeling by measurement of left ventricular torsion using speckle tracking echocardiography in patients with acute myocardial infarction. Am J Cardiol 2010; 106: 917-23.

23. Bertini M, Mollema SA, Delgado V, et al. Impact of time to reperfusion after acute myocardial infarction on myocardial demage assessed by left ventricular longitudinal strain. Am J Cardiol 2009; 104: 480-5.

24. Bolognese L, Cerisano G, Buonamici P, et al. Influence of infarct-zone viability on left ventricular remodeling after acute myocardial infarction. Circulation 1997; 96: 3353-9.

25. Orn S, Manhenke C, Anand IS, et al. Effect of left ventricular scar size, location, and transmurality on leftventricular remodeling with healed myocardial infarction. Am J Cardiol 2007; 99: 1109-14.

26. Nicolosi GL, Golcea S, Ceconi C. Effects of perindopril on cardiac remodeling and prognostic value of pre-discharge quntitative echocardiographic parameters in elderly patients after acute myocardial infarction: the PREAMI echo substudy. Eur Heart J 2009; 30: 1656-65.

27. Mattichak SJ, Harjai KJ, Dutcher JR. Left ventricular remodeling and systolic deterioration in acute myocardial infarction: findings from the Stent - PAMI study. J Inter Cardiol 2005; 18: 255-60.

28. Masci PG, Ganame J, Francone M, et al. Relationship between location and size of myocardial infarction and their reciprocal influences on post-infarction left ventricular remodelling. Eur Heart J 2011; 32: 1640-8.

29. Cerisano G, Bolognese L, Carrabba N, e al. Doppler-derived mitral deceleration time. An early strong predictor of left ventricular remodeling after reperfused anterior acute myocardial infarction. Circulation 1999; 99: 230-6.

30. Mollema SA, Liem SS, Suffoletto MS, et al. Left ventricular dyssynchrony acutely after myocardial infarction predicts left ventricular remodeling. J Am Coll Cardiol 2007; 50: 1532-40.

31. Wita K, Filipecki A, Lelek M. Prediction of left ventricular remodeling in patients with STEMI treated with primary 
$\mathrm{PCl}$ : use of quantitative myocardial contrast echocardiography. Coron Artery Dis 2011; 22: 171-8.

32. Chua S, Hung J, Chung SY, et al. Primary percutaneous coronary intervention lowers the incidence of ischemic mitral regurgitation in patients with acute ST-elevated myocardial infarction. Circ J 2010; 74: 2386-92.

33. Wita K, Berger-Kucza A, Filipecki A, et al. Predictive value of ischemic mitral regurgitation during the acute phase of ST-elevation myocardial infarction treated with primary coronary intervention for left ventricular remodeling in long-term follow-up. Coron Artery Dis 2010; 21: 325-9.

34. Araszkiewicz A, Lesiak M, Grajek S, et al. Relationship between tissue reperfusion and postinfatction left ventricular remodeling in patients with anterior wall myocardial infarction treated with primary coronary angioplasty. Kardiol Pol 2006; 64: 383-8.

35. Nicolosi GL, Golcea S, Ceconi C. Effects of perindopril on cardiac remodeling and prognostic value of pre-discharge quntitative echocardiographic parameters in elderly patients after acute myocardial infarction: the PREAMI echo substudy. Eur Heart J 2009; 30: 1656-65.

36. Cerisano G, Bolognese L, Carrabba N, et al. Doppler-derived mitral deceleration time. An early strong predictor of left ventricular remodeling after reperfused anterior acute myocardial infarction. Circulation 1999; 99: 230-6.

37. Cohn JN, Ferrari R, Sharpe N, et al. Cardiac remodeling - concepts et clinical implications: a consensus paper from an international forum on cardiac remodeling. J Am Coll Cardiol 2000; 35: 269-82.

38. Han W, Xie M, Wang XF, et al. Assesment of left ventricular torsion in patients with anterior wall myocardial infarction before and after revascularisation using speckle tracking imaging. Chin Med J (Engl) 2008; 121: 1543-8.

39. Ismail M, Khalid S, Eldemerdash $S$, et al. The percent change of strain and strain rate under dobutamine stress echocardiography predicts viability following myocardial infarction. Minerva Cardioangiol 2014 [Epub ahead of print].

40. Park YH, Kang SJ, Song JK, et al. Prognostic value of longitudinal strain after primary reperfusion therapy in patients with anterior wall acuter myocardial infarction. J Am Soc Echocardiogr 2008; 21: 262-7. 